\title{
Resurrection Isn't Everything
}

\author{
Marvin L. Birnbaum, MD, PhD
}

We are well into the decade of accountability. Like all other disciplines in Medicine, we are being called upon to define what we do and the differences we make. We are being asked, "Is it worth it?"

How will we add up? How will we judge and be judged? Up to now, the efficacy and effectiveness of what we do has been judged by the number of clinically dead persons we successfully have resurrected. And, we do it at all costs regardless of underlying problems or likelihood of a functional recovery. Survival is easy to measure and reports of enhanced survival following clinical death in the prehospital setting got prehospital emergency care started. But, we are not even sure at what point in the chain of disease care that survival should be measured. Unfortunately, we have chosen as our end point, discharge of the previously clinically dead patients from the hospital-following a hospital course with a myriad of factors over which we have little or no control or input. Other than for a few studies, we have not looked at the functional state at discharge; and the few studies that do exist do not bear well for most "successfully" resuscitated from clinical death. Once the dying process has been initiated, we remain paralyzed at reversing the relentless progression toward dysfunction associated with the development of the post-resuscitation syndromes. We really don't know much about reanimatology.

Where then do we make our greatest contributions? Daily, we impact profoundly on the continued functionality of persons who perceive they are experiencing an "emergency"; thus, the label "emergency medical services." "An emergency occurs when the victim perceives that his/her life is out of control." By definition, we respond to the person's perceived emergency - and it is not our emergency! Our mission, then, is to help persons return control to their life and to prevent further loss of control.

This may seem somewhat soft, especially if we must measure what we do. Measures to demonstrate our effectiveness in helping someone to cope with his/her emergency are more difficult than are measures of survival. And currently, other than survival, we have little upon which to base the efficacy of what we do. Efficacy means that what we do is of benefit to the patient and the society in which we practice. The effectiveness of an intervention is judged on whether, when the intervention is applied, the desired efficacy is attained. It is important in our practices to separate these definitions and use them when evaluating what we do and how we do it.

Where does this take us? How do we judge and how will we be judged? The wellworn answer is, "We prevent death and minimize morbidity." Morbidity must be defined. Perhaps, it has been summed-up best by Daniels: we help people return to normal species functioning and optimize their opportunity range. But this sounds high-falutin' and seems impossible to assess. How can we state that we minimized morbidity-when, if we are effective, it doesn't happen?

The efficacy of prehospital EMS and medicine rests in this elusive arena. We know we prevent cardiac arrest in patients with substernal chest pain and PVCs, but how do we know this patient was one of the $60 \%$ who would have died within the first hour of the onset of symptoms. We know we make a difference for the patient who has sustained a cervical fracture and, because of our interventions, does not transect her spinal cord. We know we make a difference in the patient who is hypoglycemic. We 


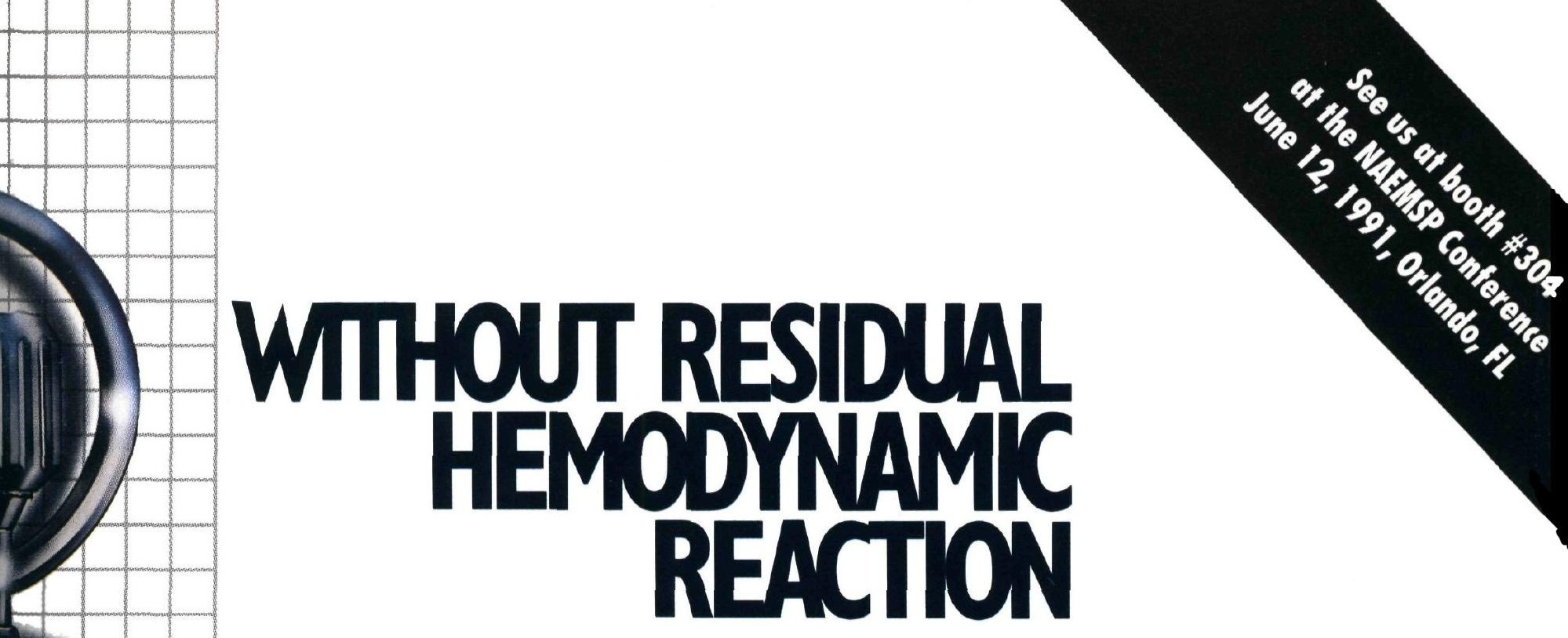

ADENOCARD has an unsurpassed safety profile. , $2^{2}$ With a half-life estimated to be less than 10 seconds, adverse reactions are minimal and of brief duration. And, ADENOCARD has no effect on subsequent cardiovascular therapies.
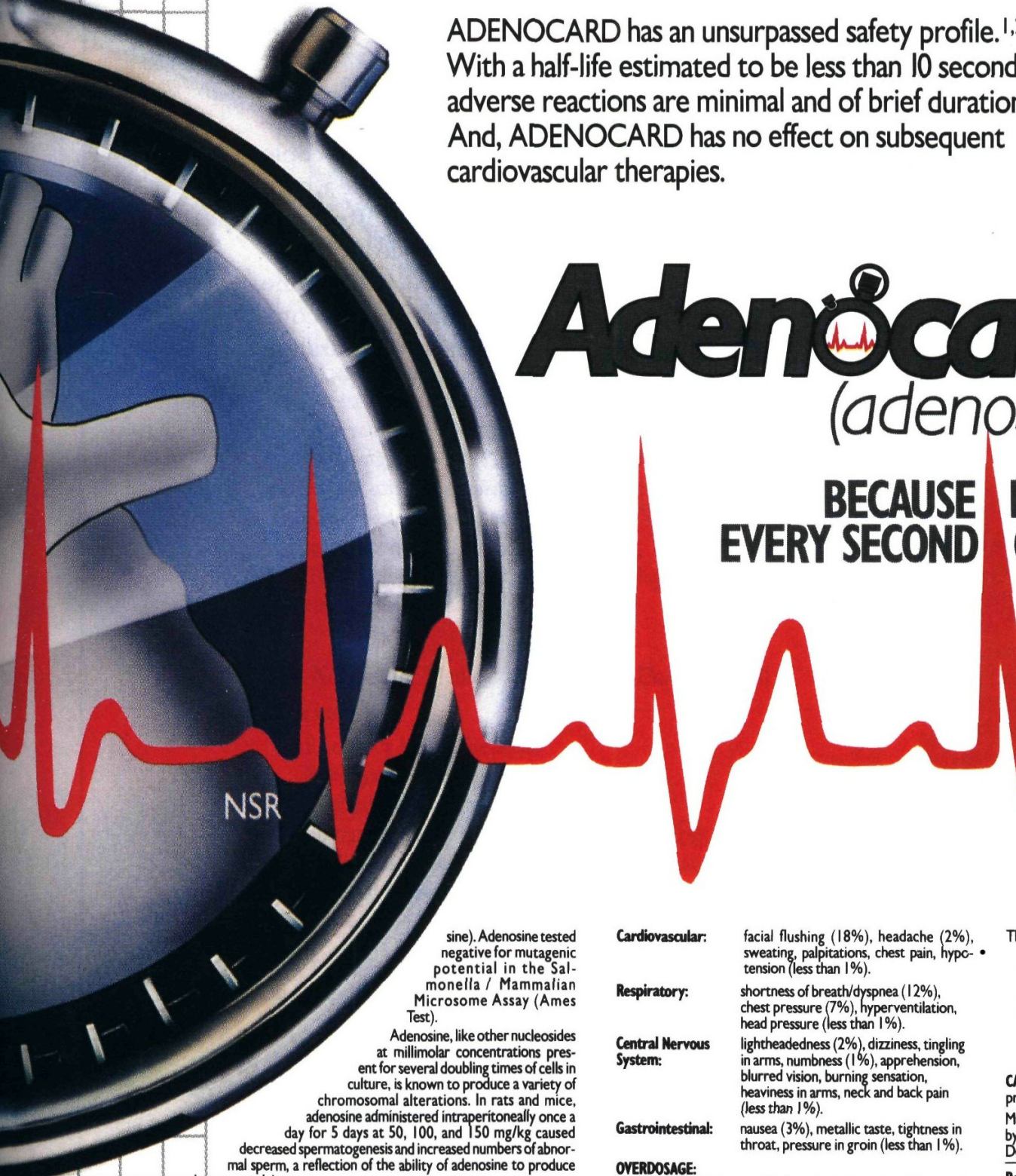

- Category C:Animal reppoduction studies have not been conducted with adenosine; nor have studies been performed in pregnant women. As adenosine is a naturally occurring material, widely dispersed throughout the body no fetal effects would be anticipated. However, since it is not known wether Adenocard can cause feat harm when administered to pregnant women. Adenocard should be used during pregnancy only if clearly needed

The half-life of Adenocard (adenosine) is less than 10 seconds. Thus, adverse effects are generally rapidly self-limiting. Treatment of any prolonged adverse effects should be individualize ward the specific effect. Methylxanthines such as caffeine and theophylline, are competitive antagonists of
adenosine.

\section{DOSAGE AND ADMINISTRATION:}

Adenocard (adenosine) Injection should be given as a rapid bolus intravenous injection. To be certain the solution reaches the systemic circulation, it should be administered either as proximal as possible and followed by a rapid saline lush. as proximal as possible and followed by a rapid saline llush.

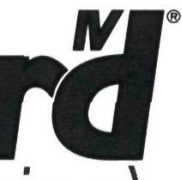

sine)

N PSVT COUNTS

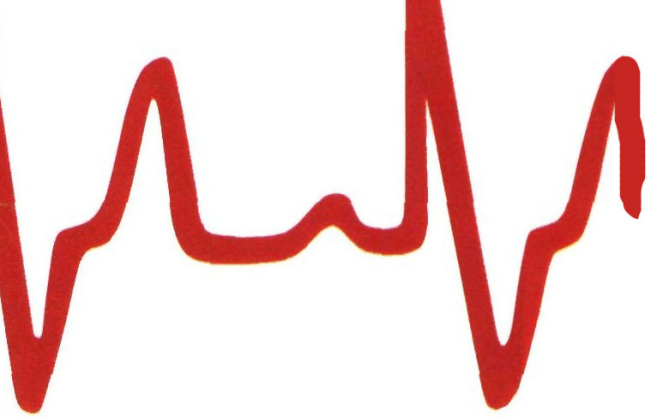


know we make a difference for the person suffering hypovolemic shock. But these ministrations really are preventative, and how do we know he/she would have crashed if we hadn't been there?

We need to devise some ways to demonstrate our impact on these patients. Unfortunately, controlled experiments in these areas are not possible. But, there are ways to measure the impact of our interventions and achievement of beneficial outcomes. First, we must identify areas in which we believe we make a difference (produce benefits). Second, we must develop tools that are sufficiently sensitive to show changes in patient conditions during the short time we spend with them; we could demonstrate changes in severity for specific conditions. For example, we could evaluate changes in the Glascow Coma Score during our contact with a patient with an altered conscious state. We could demonstrate changes in the severity of a shock state using the American College of Surgeons' classification of severity of shock. These are severity scoring systems, and we must focus on their use. Third, we must define standards of practice in these specific are- nas. Thus, we could judge and be judged according to established standards. Once standards of practice for little bits and pieces of what we do are established, criteria by which to judge our effectiveness in achieving these standards can be developed. If we are to become a well-defined part of medicine, we must define our standards. Each organization sponsoring this Journal has a major role in developing minimum standards and the criteria by which to judge our achievement of these standards. Minimum Standards must be stated for specific areas of practice. We must concentrate on welldefined bits of our practice.

We must come away from concentrating on how many clinically dead people we resurrect and begin to define where and how we really make a difference. The establishment of standards of practice and of criteria by which to define our ability to attain them really comprise the mission of each organization for this decade of accountability.

\section{Reference:}

1. Personal communication with Barb Bina, 1979. 


\section{Mosby - Year Book and NAEMSP offer the Gold Standard in EMS Medical Direction}

The EMS Medical

Directors' Handbook

\section{Edited by Alexander E. Kuehl, M.D.}

with contributions by over 30 national EMS leaders.

The all-in-one source for understanding the intricacies of EMS systems and medical direction, and finding solutions to your system woes.

The Handbook, the basis of the NAEMSP's National EMS Medical Directors' Course, is a concise overview of EMS system components, their design, implementation and operation. Learn:

- How strong medical involvement will help your system meet the public's expectations:

- The basics of direct and indirect medical control;

- How your system interfaces with law, politics and the rest of the medical community;

- How to effectively supervise all types of EMS systems;

- Clear objectives for improving prehospital care, from system development to operational issues;

- Timely information on funding strategies, legislation, labor relations, ethical issues and more.

Not just for Medical Directors, the Handbook will also benefit:

- Emergency Medical Residents

- EMS System Administrators

- Paramedic Supervisors

- Emergency Nurses

- Quality Assurance Coordinators

- EMS and Public Health Students

Copyright 1989

448 pages

Book Code 05854

Mosby - Year Book is also pleased to be chosen by NAEMSP to publish

\section{Quality Assurance in Prehospital Care}

Edited by Robert A. Swor, D.0.

with contributions by more than 20 EMS leaders

Successful quality assurance programs ensure the ongoing safe, ethical, clinically sound and cost-effective practice of medicine in the field. Providers at all levels are given the keys to establishing and maintaining such programs, with emphasis on the methodologies of problem solving and practical application of solutions.

Publishing October 1992
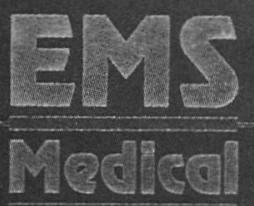

Wh

B) $2600 \mathrm{H}$

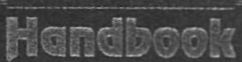

19.

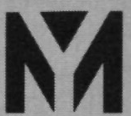

Mosby Year Book

7250 Parkway Dr. Suite 510 Hanover, MD 21076

Also of interest...

ACLS: Certification Preparation and a

Comprehensive Review, 2nd Ed.

Ken Grauer, M.D., FAAFP

Book Code: 01751

ACLS: Mega Code Review Study Cards Ken Grauer, M.D., FAAFP

Book Code: 02937

Disaster Response: Principles of Preparation and Coordination

Erik Auf der Heide, M.D.

Book Code: 00385

Treatment Protocols \& Algorithms for

Prehospital Care

J. Thomas Ward Jr., M.D.

Book Code: 05321

Advanced Medical Life Support: Adult

Medical Emergencies

Mikel A. Rothenberg, M.D.

Book Code: 04284

Prehospital Emergency Drugs Pocket Reference Kevin G. Madiagn, CCFP Book Code: 03375

These titles are also available in the Jems Bookstore.

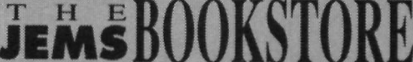

To order, call 800/266-JEMS 
The MFDICAL 911 Sourcebooks

When you're searching for hard-to-find EMS information, these are the reference books to have. They scan, sort and digest a mountain of material, saving your valuable time.

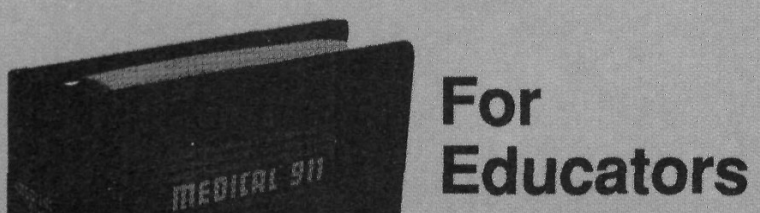

There's a wealth of information available to EMS educators, and this binder helps you find the best of it quickly and efficiently.

Build a course, or a whole curriculum, with the comprehensive

MEDICAL 911 Educator Sourcebook as

\section{your guide!}

Find relevant articles fast. The "EMS Literature" section provides over 2100 journal citations, using 20 convenient topic categories.

Speed-read the library. The "EMS Books" section lists nearly 800 titles covering all aspects of emergency medical services.

Make the most of A-V. The "EMS Instructional Materials" section contains an up-to-date compilation of over 500 audiovisual aids, including videos, films and slides. (You also get listings of training programs, plus sources for simulated injury kits and other teaching materials.)

Add "high tech" approaches. The "EMS Software and Online Databases" section tells you about computer-aided research, and about computer programs useful for instruction.

$\$ 65$

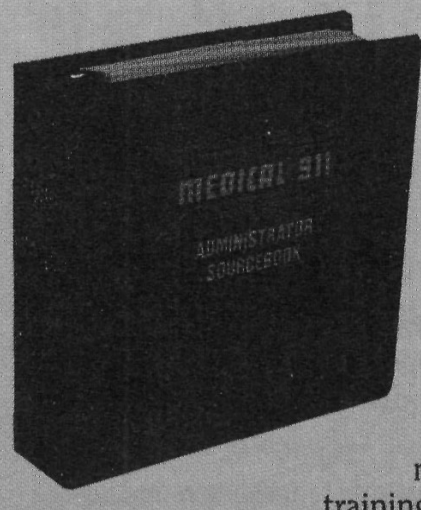

For Administrators

Successful administrators make good decisions by staying informed, and by knowing where to find up-to-date information.

What does the EMS literature say about certain patient care techtraining methods?

When you need to know, there's a way to find out: the MEDICAL 911 Administrator Sourcebook!

Discover who handles what. The "EMS Agencies and Organizations" section lists more than 45 groups, with summary information and contacts.

Avoid reinventing the wheel. The "EMS Reports" section covers all the major topics and brings you over 300 abstracts, along with details on how to get the complete reports.

Do your homework the smart way. The "EMS Surveys" section recaps key results from important surveys, and shows you where you can get additional information.

Experts on call. The "EMS Consulting Services" section lets you connect with top consultants and lawyers-specialists in issues relating to emergency medical services.

Get a great guide to suppliers. The "EMS Products and Services" section has over a thousand entries, with vendor names and telephone numbers.

$\$ 125$

\section{Buy Both...Save \$25 and get a big bonus!}

Interested in both educational and administrative issues? For just $\$ 165-\$ 25$ off the combined individual prices - you can own both Sourcebooks. And by ordering both now, you also get a $\$ 49$ value FREE bonus: a one year subscription to our monthly newsletter, the EMS Insider.

\section{FREE UPDATE SERVICE}

Material in the MEDICAL 911 binders is always being revised and updated. When you buy either volume, or both, you get $F R E E$ update service for one year from date of purchase!

\section{Jems Communications GUARANTIEA}

If you're not completely satisfied with your MEDICAL 911 Sourcebook(s), return within 30 days for a full refund.

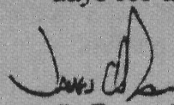

James O. Page, Executive Director Emergency Care Information Center

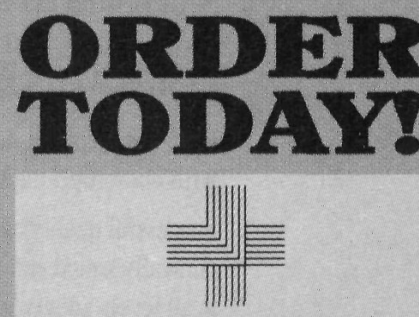

EMERGENCY CARE INFORMATION CENTER

(800) 266-JEMS 